\title{
Gas and dust in the inner disk of the Herbig Ae star MWC 758
}

\author{
A. Isella ${ }^{1,2}$, E. Tatulli ${ }^{1,3}$, A. Natta ${ }^{1}$, and L. Testi ${ }^{1,4}$ \\ 1 Osservatorio Astrofisico di Arcetri, INAF, Largo E. Fermi 5, 50125 Firenze, Italy \\ 2 California Institute of Technology, MC 105-24, 1200 East California Blvd., Pasadena CA 91125, USA \\ e-mail: isella@astro.caltech.edu \\ 3 Laboratoire d'Astrophysique de Grenoble, UMR 5571, Université Joseph Fourier/CNRS, BP 53, 38041 Grenoble Cedex 9, France \\ ${ }^{4}$ ESO, Karl-Schwarschild Strasse 2, 85748 Garching bei München, Germany
}

Received 24 February 2008 / Accepted 25 March 2008

\section{ABSTRACT}

\begin{abstract}
We investigate the origin of the near-infrared emission of the Herbig Ae star MWC 758 on sub-astronomical unit (AU) scales using spectrally dispersed low resolution $(R=35)$ AMBER/VLTI interferometric observations in the $H(1.7 \mu \mathrm{m})$ and $K(2.2 \mu \mathrm{m})$ bands. We find that the $K$ band visibilities and closure phases are consistent with the presence of a dusty disk inner rim located at the dust evaporation distance $(0.4 \mathrm{AU})$ while the bulk of the $H$ band emission arises within $0.1 \mathrm{AU}$ from the central star. Comparing the observational results with theoretical model predictions, we suggest that the $H$ band emission is dominated by an hot gaseous accretion disk.
\end{abstract}

Key words. accretion, accretion disks - techniques: interferometric - stars: planetary systems: protoplanetary disks

\section{Introduction}

The development of long baseline near-infrared (NIR) interferometry allows us to spatially resolve the emission of the innermost regions of disks around young pre-main sequence stars ( $\lesssim 1 \mathrm{AU}$ in the nearest star forming regions). These regions are important for an understanding of the star-disk interaction, the gas accretion process onto the star, and the launching of jets and winds. Moreover, planet formation is likely to be favored in the high density regions of the inner disk.

The existing NIR interferometric observations of low mass $\mathrm{T}$ Tauri and intermediate mass Herbig Ae disks (HAe hereafter; Millan-Gabet et al. 2007; Monnier et al. 2006; Akeson et al. 2005; Eisner et al. 2004, 2007) show that emission in the $K$ band $(2.2 \mu \mathrm{m})$ is dominated by dust at the sublimation temperature, located in a puffed-up rim (Dullemond et al. 2001; Isella \& Natta 2005). For the more luminous HBe stars, dust emission alone cannot explain the observed visibilities, and it has been suggested that the $K$ band excess flux is dominated by the emission of a dense, optically thick gaseous disk which extends well inside the dust sublimation radius (Malbet et al. 2007). Hints of the presence of hot gaseous disk are also provided by recent spectrally dispersed low resolution observations of a sample of HAe stars where dust emission alone cannot reproduce the spectral variation of the visibility across the $K$ band (Eisner et al. 2007). Most gas tracers, such as the hydrogen recombination lines, are very likely emitted by matter which is either accreting or ejected from the system (Malbet et al. 2007; Tatulli et al. 2007; Kraus et al. 2008; Eisner et al. 2007b), rather than from the gaseous disk itself. In only one case (51 Oph; Tatulli et al. 2008) do Amber/VLTI medium spectral resolution

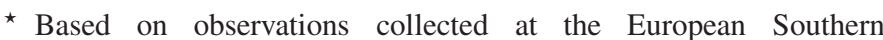
Observatory, Chile, Program 078.C-0243.
}

observations of the $\mathrm{CO} v=2-0$ lines at $2.3 \mu \mathrm{m}$ confirm their origin in the inner disk, inside the dust sublimation radius.

In this Letter we present spectro-interferometric observations of the HAe star MWC 758 (ST A5, D $=200 \mathrm{pc}$, $\left.L_{*}=22 L_{\odot}, M_{*}=2 M_{\odot}\right)$ across the $H$ and the $K$ band obtained with AMBER/VLTI. We show that the $H$ and $K$ band excess emission arises from two different disk regions, the first of which is much more compact and hotter than the second. We identify the dominant source of the $2 \mu \mathrm{m}$ emission as the dust rim at the sublimation radius, and the dominant source of the $1.7 \mu \mathrm{m}$ emission as the gaseous disk inside this radius.

The structure of this letter is as follows. Section 2 describes the observations and the data reduction, Sect. 3 describes the results, which are discussed in Sect. 4. Section 5 summarizes our conclusions.

\section{Observations and data reduction}

MWC $758(K=5.8 \mathrm{mag}, H=6.5 \mathrm{mag})$ was observed with AMBER on December 30, 2006, and on January 8, 2007, with three $8 \mathrm{~m}$ Unit Telescopes (UTs) of the VLTI, using the UT1UT3-UT4 triplet. On both dates, the source was observed close to transit; the hour angles ( 0.25 and $0.54 \mathrm{~h}$, respectively) of the two sets of observations were separated by $20 \mathrm{~min}$. At the time of the observations, the parameters of the three projected baselines were: $B=81 \mathrm{~m}$, position angle PA $=45^{\circ}$ (UT1-UT3), $B=59 \mathrm{~m}, \mathrm{PA}=102^{\circ}$ (UT3-UT4), and $B=123 \mathrm{~m}, \mathrm{PA}=69^{\circ}$ (UT1-UT4), on Dec. 30, 2006, and $B=84 \mathrm{~m}, \mathrm{PA}=45^{\circ}$ (UT1UT3), $B=58 \mathrm{~m}, \mathrm{PA}=101^{\circ}$ (UT3-UT4), and $B=125 \mathrm{~m}$, $\mathrm{PA}=68^{\circ}$ (UT1-UT4), where the PA is measured east of north. Observations were performed in low spectral resolution mode $(R=35)$, in both the $H$ and $K$ bands.

Raw visibilities and closure phases as a function of wavelength were computed following the standard data reduction algorithms described in Tatulli et al. (2007), separately for each 


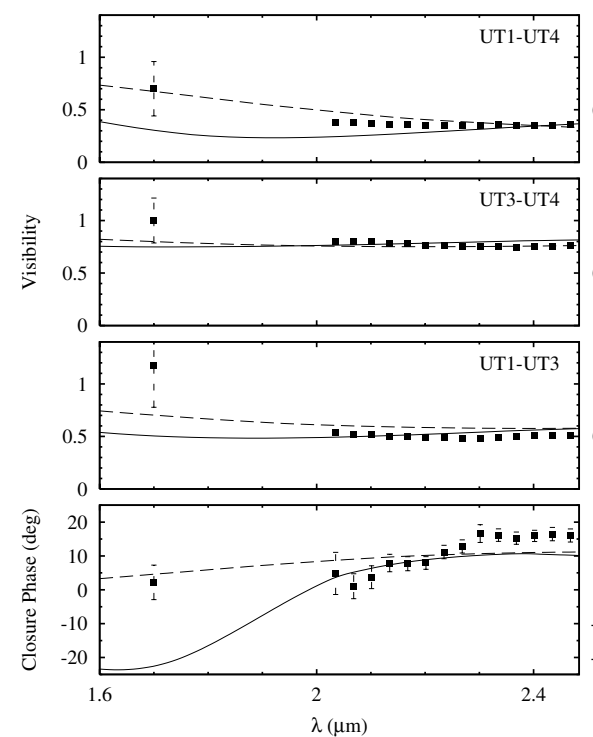

(a)
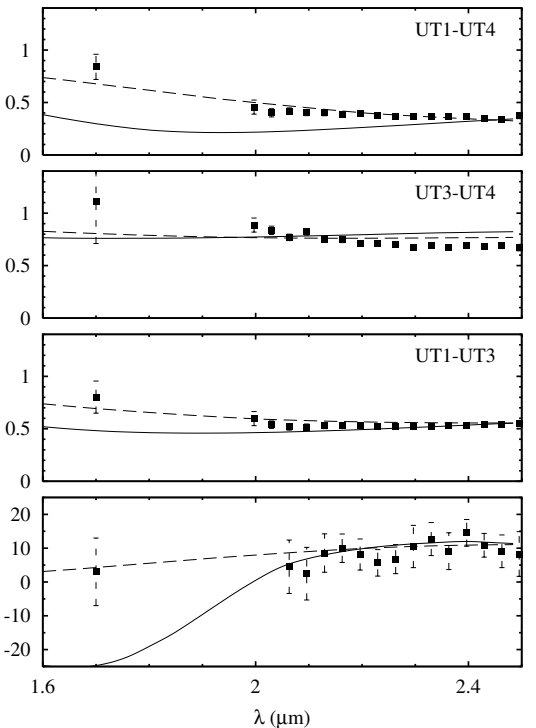

(b)

Fig. 1. Spectrally dispersed visibility and closure phase measured across the $H(1.65 \mu \mathrm{m})$ and $K(2.2 \mu \mathrm{m})$ bands. Panels a) and b) show the data for two different hour angles, $\mathrm{HA}=0.25 \mathrm{~h}$ and $\mathrm{HA}=0.54 \mathrm{~h}$ respectively. The three upper panels show calibrated visibilities for the three different baselines as labeled in the figure and described in Sect. 2; corresponding closure phases are plotted in the bottom panel. Visibility errors across $K$ band are between 3 and 5\%; $H$ band data has been spectrally averaged to increase signal to noise ratio, the associated error is about $30 \%$. Solid lines correspond to the rim model (I), dashed lines to the gas+rim model (II). Model parameters are reported in Table 1.

spectral band. The raw visibilities were corrected for atmospheric and instrumental effects by dividing them by the raw visibilities measured on a reference source. As a calibrator, we chose HD 34053 ( $K=5.9, H=5.9$ ) which has similar magnitudes and, according to the CADAR catalog (Pasinetti Fracassini et al. 2001), has a diameter of 0.2 mas - that is, unresolved by the interferometer. The closure phase is theoretically independent of the Earth's atmosphere (see, e.g., Monnier et al. 2007, for the definition of closure phase) and does not need to be calibrated. However, AMBER introduces an offset in the closure phase which is corrected by subtracting the calibrator closure phase from the MWC 758 observed values.

\section{Results}

The results are presented in Fig. 1, which shows the visibilities and closure phases as a function of wavelength for the two different data sets: panel (a) refers to the Dec. 30, 2006 observations (hour angle HA $=0.25 \mathrm{~h}$ ), panel (b) to the Jan. 8, 2007 one $(\mathrm{HA}=0.54 \mathrm{~h})$. The results from the two data sets almost coincide, as expected given the small difference in HA. Due to the lower flux in the $H$ band, data between 1.6 and $1.8 \mu \mathrm{m}$ are averaged in order to increase the signal to noise ratio. Even so, the error on the $H$ band visibility is about $30 \%$, much larger than that of the $K$ band data ( $~ 5 \%)$.

In the $K$ band, the source is resolved on all the baselines, which provide an angular resolution of 1.8 mas, corresponding to $0.35 \mathrm{AU}$ at the distance of MWC 758. The closure phase is always larger than zero and increases with wavelength between 2 and $2.5 \mu \mathrm{m}$. Note that a non zero closure phase implies that the $K$ band emission is not centrally symmetric. This measurement alone rules out models such as spherical envelopes, flat disks, etc. for the $K$ band emission (see also the discussion in Monnier et al. 2006).

In the $H$ band, the MWC 758 emission is unresolved on the UT3-UT4 and UT1-UT3 baselines, with a visibility equal
Table 1. Parameters of the best fit models shown in Figs. 1 and 2. Model I corresponds to the rim model described in Isella \& Natta (2005); in Model II we have added to the star and rim an additional source of emission, as described in the text.

\begin{tabular}{llll}
\hline \hline & gas emission & dust emission & \\
\hline & & $T_{\text {eff }} \sim 1400 \mathrm{~K}$ & incl $=40^{\circ}$ \\
& & $R_{\text {in }}=0.34 \mathrm{AU}$ & \\
I & & $R_{\text {out }}=0.39 \mathrm{AU}$ & \\
& & $X_{H}=0.50$ & \\
& & $X_{K}=0.79$ & \\
\hline & $T_{\text {eff }}=2500 \mathrm{~K}$ & $T_{\text {eff }} \sim 1300 \mathrm{~K}$ & incl $=30^{\circ}$ \\
& $R_{\text {in }} \gtrsim 0.05 \mathrm{AU}$ & $R_{\text {in }}=0.40 \mathrm{AU}$ & \\
II & $R_{\text {out }} \leqslant 0.1 \mathrm{AU}$ & $R_{\text {out }}=0.49 \mathrm{AU}$ & \\
& $X_{H}=0.41$ & $X_{H}=0.18$ & \\
& $X_{K}=0.33$ & $X_{K}=0.46$ & \\
\hline & & &
\end{tabular}

to 1 within the error bars; on the longest baseline UT1-UT4 the visibility is slightly smaller than unity for both data sets ( $V=0.8 \pm 0.1$ ). The $H$ band closure phase is consistent with 0 , as expected given that the emission is almost unresolved.

\section{Discussion}

To analyze the AMBER data, we compute visibilities and closure phases as a function of wavelength using the rim model of Isella \& Natta (2005), where a complete description of the physical models and of their limitations can be found. Once the stellar parameters are defined, the rim location and shape depend only on the grain emissivity, i.e., for known dust composition, on the grain size distribution. We assume silicate grains, which are the grains with the highest sublimation temperature according to Pollack et al. (1994). The fitting procedure is described in Isella et al. (2006) and the rim parameters that give the best fit to the data are given in Table $1 . R_{\text {in }}$ is the distance of the rim 


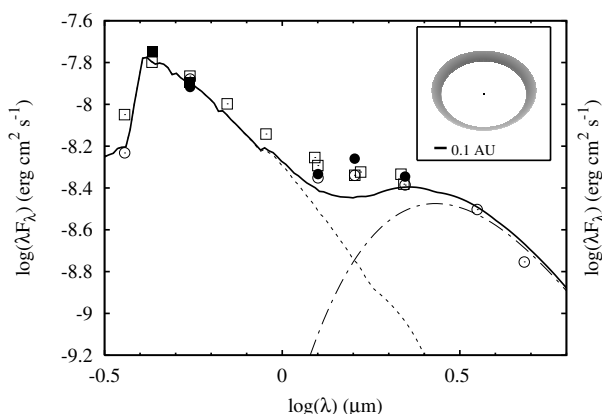

(a)

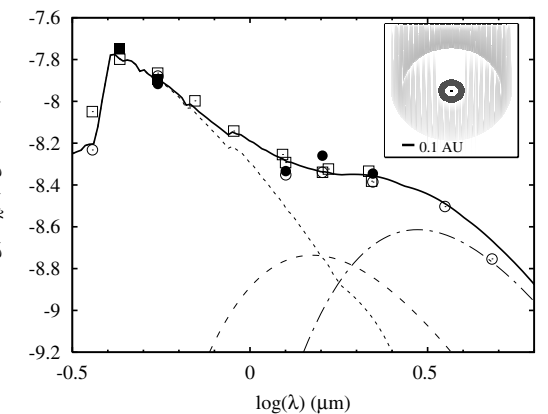

(b)

Fig. 2. SED of MWC 758. Symbols show the photometry from the literature corrected for an extinction $A_{V}=0.4, R=3.1$. The stellar spectrum (short-dashed line) is that of a Kurucz model with $T_{\text {eff }}=8000, \log g=4$. Panel a) shows the flux predicted by model I (solid line) composed of the dusty disk rim emission (short-long dashed line) and the star. Panel b) shows the prediction for model II where an additional black body component is added (dashed line). In each panel the image of the corresponding model is shown in the inset. Refer to Table 1 for model parameters.

from the star on the disk midplane, $R_{\text {out }}$ the distance of the top of the rim, so that $\Delta R=R_{\text {out }}-R_{\text {in }}$ measures the curvature of the rim due to the dependence of the dust sublimation temperature on the gas density. The rim emission can be approximately described as a black-body at $T_{\text {eff. The two quantities }} X_{H}$ and $X_{K}$ give the fraction of the dusty rim and the gaseous disk emission (see the discussion below) to the total flux in the $H$ and $K$ band respectively; the flux missing to reach unity is due to the stellar photosphere.

\subsection{Star + rim models}

Model I includes the star and the rim emission only. As shown in Fig. 1 (solid curves), it provides an adequate, although not perfect, description of the $K$ band visibility and closure phase measurements. Both the derived rim inner radius $(0.34 \mathrm{AU})$ and the disk inclination $\left(40^{\circ}\right)$ are in agreement with the values obtained in Isella et al. (2006) based on earlier PTI observations; the disk position angle is not well constrained by our data, varying between $90^{\circ}$ and $150^{\circ}$.

However, such models fail to account for the observations in the $H$ band, where they predict that the total emission should be spatially resolved. The reason is clear if we consider that the rim is physically narrow, e.g. $\Delta R \sim 0.05 \mathrm{AU}$, and has practically the same size at all wavelengths. In this case, a variation of visibility and closure phase with wavelength may occur for two reasons only, one intrinsic, because the contribution of the unresolved stellar flux increases at shorter wavelengths, and one instrumental, since the interferometer resolution decreases with wavelength. In the calculation of the visibility these two effects tend to compensate for one another and the resulting values are roughly constant, contrary to what we observe. The discrepancy between observations and model predictions is even more striking if we consider the dependence of the closure phase on wavelength. The higher angular resolution achieved in $H$ band amplifies the asymmetry of the model emission, resulting in very large values of the closure phase in the $H$ band, contrary to what is observed. Note that a discrepancy between the rim model and the observations is also present in the SED (Fig. 2 panel $(a)^{1}$ ) since the best fit rim model can account only for about $80 \%$ of the emission measured in the $H$ band.

1 Photometry from Eisner et al. (2004), Malfait et al. (1998), Beskrovnaya et al. (1999), Cutri et al. (2003).

\subsection{Evidence for gas emission in the inner disk}

Motivated by these considerations, we modified our rim model introducing an additional source of emission, which we describe as a uniform black body ring where the ring radii and its effective temperature are free parameters. The values of the parameters of the best-fitting model (Model II) are given in Table 1. As shown in Figs. 1 and 2 (dashed lines) a good fit to both the $H$ and $K$ band visibilities and fluxes is obtained for $T_{\text {eff }}=2500 \mathrm{~K}$ and a ring outer radius $\lessgtr 0.1 \mathrm{AU}$. The exact values of the inner and outer ring radius are not well constrained since the $H$ band emission is poorly resolved. However, the lower $H$ band visibility measured on the longest baseline seems to indicate ring radii between 0.05 and $0.1 \mathrm{AU}$. Figure $2 \mathrm{~b}$ shows that this model fits the MWC 758 SED very well. Such a model is clearly a simplified representation of a more realistic inner disk in which the gas temperature and emission decrease smoothly with increasing stellocentric distances. Nevertheless, it can reproduce the rough spatial scale for the gas emission and it is therefore adequate to understand the current observations.

The physical origin of this additional emission is hard to constrain from the available data. We suggest that it can be identified with the emission of the gaseous accretion disk inside the dusty rim, based on the comparison of the observational constraints we have with the predictions of the Muzerolle et al. (2004) models. These authors model the physical structure of the inner disk of a typical Herbig Ae star, including dust evaporation and formation of the rim. Inside the rim, the gaseous disk properties depend on the mass accretion rate $\dot{M}_{\text {acc }}$. We estimate that in MWC $758 \dot{M}_{\text {acc }} \sim 2 \times 10^{-7} M_{\odot} / \mathrm{yr}$, based on the strength of the $\mathrm{Br} \gamma$ emission $^{2}$, as described in Garcia Lopez et al. (2006). For such an $\dot{M}_{\text {acc }}$, the models predict that the gas in the disk mid plane reaches a temperature of about $2500 \mathrm{~K}$ at a distance of $0.1 \mathrm{AU}$, consistent with our constraint that the hot component is located within $0.1 \mathrm{AU}$ of the star. Figure 3 shows the emission of the gas disk for two accretion rates $\left(10^{-7}\right.$ and $\left.10^{-6} M_{\odot} / \mathrm{yr}\right)$, which encompass the estimated value in MWC 758. The $2500 \mathrm{~K}$ black body emission is roughly intermediate between the two, and its spectral shape is similar, supporting the interpretation of the "second ring" as gas emission within the dust sublimation radius.

The Muzerolle et al. (2004) models have been computed for slightly different stellar parameters, so that the comparison is necessarily very qualitative. Also, we were not able to

\footnotetext{
2 Telescopio Nazionale Galileo unpublished data.
} 


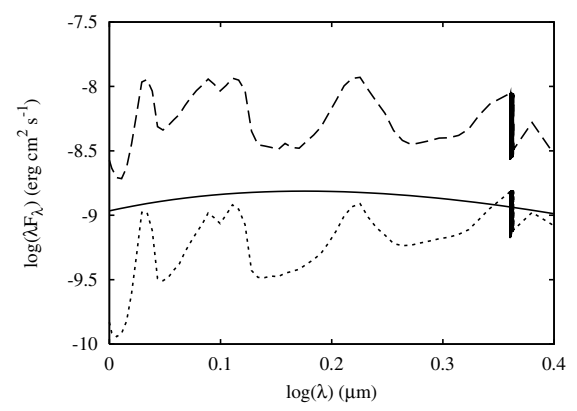

Fig. 3. Comparison of the $2500 \mathrm{~K}$ black body emission, added in model II (solid line), with the emission of the inner gaseous disk for two mass accretion rates, $10^{-6} M_{\odot} / \mathrm{yr}$ (top curve) and $10^{-7} M_{\odot} / \mathrm{yr}$ (lower curve), over the relevant range of wavelength between 1 and $2.5 \mu \mathrm{m}$ respectively.

compute the expected visibilities and phase closures of the Muzerolle et al. (2004) models, since only the wavelength dependence of the spatially integrated flux is available.

The introduction of the additional black body emission has consequences for the rim structure and for the derived dust grain size. In particular, going from model I to model II, the inner rim radius increases from $0.34 \mathrm{AU}$, obtained with a dust grain size of $1 \mu \mathrm{m}$, to $0.40 \mathrm{AU}$ corresponding to dust grains of $0.5 \mu \mathrm{m}$. This confirms (Isella et al. 2006) that the grains in the inner disk of MWC 758 are on average larger than the typical dust size in the interstellar medium, i.e. $0.01 \mu \mathrm{m}$. However, an accurate determination of the grain properties requires us to understand the global properties of the inner disk, gas and dust.

\section{Conclusions}

We present spectro-interferometric observations $(R=35)$ of the pre-main sequence HAe star MWC 758, obtained with VLTI/Amber in the $H$ and $K$ bands. The observations have been obtained with three UT telescopes in order to measure both spectrally dispersed vibilities and closure phases. The $H$-band data and the closure phase are essential quantities to distinguish between different models of the disk structure. The observations provide a spatial resolution of 0.8 mas in the $H$ band and 1.8 mas in the $K$ band, corresponding to scale sizes of 0.16 and $0.35 \mathrm{AU}$, at the distance of MWC 758.

In the $K$ band, the source is resolved with non-zero closure phase (about $10^{\circ}$ ), as expected if the emission is due to dust in a rim at the sublimation radius ( $0.49 \mathrm{AU})$, seen at an inclination of about $30^{\circ}$. In the $H$ band the source is only barely resolved with the longest baseline (125 m), implying that the emission at these wavelengths arises within $0.1 \mathrm{AU}$ of the central star.
We suggest that moving in wavelength from $\sim 2.5 \mu \mathrm{m}$ to the $H$ band, centered at $1.7 \mu \mathrm{m}$, one moves from rim-dominated emission to a hotter, more compact component which is qualitatively consistent with the emission of the dust-free inner gaseous disk, as modeled by Muzerolle et al. (2004). These results support the suggestion of Eisner et al. (2007) that an extra component in addition to the dust would improve the fit to the spectrally dispersed, low resolution $K$ band visibilities obtained with the Palomar Testbed Interferometer for a small sample of HAe stars.

Our results show that the innermost gaseous disk can be detected and studied with current NIR interferometers through the detection of the continuum gas emission in the $H$ band, where the gas contribution is higher than the stellar and dust emission, which dominate at shorter and longer wavelengths, respectively. Acccurate models of the spatial and spectral dependence of the inner gaseous disk emission for a range of stellar and accretion properties are much needed.

Acknowledgements. We thank Paola d'Alessio and James Muzerolle for providing as with the gas SED models shown in Fig. 3. This project was partially supported by the INAF 2005 grant "Interferometria infrarossa: ottimizzazione di osservazioni astrofisiche" and by the INAF 2006 grant "From Disks to Planetary Systems". This work was performed in part under contract with the Jet Propulsion Laboratory (JPL) funded by NASA through the Michelson Fellowship Program. J.P.L. is managed for NASA by the California Institute of Technology.

\section{References}

Akeson, R. L., Boden, A. F., Monnier, J. D., et al. 2005, ApJ, 635, 1173 Beskrovnaya, N. G., Pogodin, M. A., Miroshnichenko, A. S., et al. 1999, A\&A, 343,163

Brittain, S. D., Simon, T., Najita, J. R., et al. 2007, ApJ, 659, 685

Cutri, R. M., et al. 2003, The IRSA 2MASS All-Sky Point Source Catalog, NASA/IPAC Infrared Science Archive

Dullemond, C. P., Dominik, C., \& Natta, A. 2001, ApJ, 560, 957

Eisner, J. A. 2007, Nature, 447, 7144

Eisner, J. A., Lane, B. F., Hillebrand, L. A., et al. 2004, ApJ, 613, 1049

Eisner, J. A., Chiang, E. I., Lane, B. F., \& Akeson, R. L. 2007, ApJ, 657, 347

Garcia Lopez, R., Natta, A., Testi, L., \& Habart, E. 2006, A\&A, 459, 837

Isella, A., \& Natta, A. 2005, A\&A, 438, 899

Isella, A., Testi, L., \& Natta, A. 2006, A\&A, 451, 951

Malbet, F., Benisty, M., de Wit, W.-J., et al. 2007, A\&A, 464, 43

Malfait, K., Bogaert, E., \& Waelkens, C. 1998, A\&A, 331, 211

Millan-Gabet, R., Malbet, F., et al. 2007, Protostars and Planets V, ed. B.

Reipurth, D. Jewitt, \& K. Keil (Tucson: University of Arizona Press), 539

Monnier, J. D., Berger, J.-P., Millan-Gabet, R., et al. 2006, ApJ, 647, 444

Muzerolle, J., D’Alessio, P., Calvet, N., \& Hartmann, L. 2004, ApJ, 617, 406

Najita, J. R., et al. 2007, Protostars and Planets V, ed. B. Reipurth, D. Jewitt, \&

K. Keil (Tucson: University of Arizona Press), 507

Pasinetti Fracassini, L. E., Pastori, L., Covino, S., \& Pozzi, A. 2001, A\&A, 367, 521

Pollack, J. B., Hollenbach, D., Beckwith, S., et al. 1994, ApJ, 421, 615

Tatulli, E., Millour, F., Chelli, A., et al. 2007, A\&A, 464, 29 